hep-ph/yymmdd

FTUV/98-100

IFIC/98-101

\title{
Neutrinoless double beta decay in Supersymmetry with bilinear R-parity breaking
}

\author{
M. Hirsch ${ }^{1}$ and J. W. F. Vallef \\ Instituto de Física Corpuscular - C.S.I.C. \\ Departamento de Física Teòrica, Universitat of València \\ 46100 Burjassot, València, Spain \\ http://flamenco.uv.es/d
}

PACS: 12.60Jv, 14.60Pq, 23.40-s

\begin{abstract}
We reanalyze the contributions to neutrinoless double beta $\left(\beta \beta_{0 \nu}\right)$ decay from supersymmetry with explicit breaking of R-parity. Although we keep both bilinear and trilinear terms, our emphasis is put on bilinear R-parity breaking terms, because these mimic more closely the models where the breaking of R-parity is spontaneous. Comparing the relevant Feynman diagrams we conclude that the usual mass mechanism of double beta decay is the dominant one. From the non-observation of $\beta \beta_{0 \nu}$ decay we set limits on the bilinear R-parity breaking parameters of typically a (few) $100 \mathrm{keV}$. Despite such stringent bounds, we stress that the magnitude of R-parity violating phenomena that can be expected at accelerator experiments can be very large, since they involve mainly the third generation, while $\beta \beta_{0 \nu}$ decay constrains only the first generation couplings.
\end{abstract}

\footnotetext{
${ }^{1}$ mahirsch@neutrinos.uv.es

2valle@neutrinos.uv.es
} 


\section{Introduction}

The most well-studied realization of supersymmetry has a conserved R-parity. This is a multiplicative quantum number which can be defined as $R_{P}=$ $(-1)^{3 B+L+2 S}$, where $B$ and $L$ are the baryon and lepton number and $S$ the spin of the corresponding particle. This defining property of the Minimal Supersymmetric Standard Model (MSSM) [1] is theoretically ad hoc since the origin of R-parity conservation is unknown. Since the early days it was recognized that the violation of R-parity could arise as residual effects in larger theories [2] or spontaneously due to a sneutrino vacuum expectation value (VEV) [3]. We find the models with pure spontaneous breaking more well-motivated than explicit breaking models, in the sense that they put R-parity breaking on a dynamical footing. They have only lepton number violating $(\not L)$ terms and are therefore in agreement with proton stability. The simplest models of this type (with the $S U(2) \otimes U(1)$ gauge structure) are characterized by the existence of a physical Goldstone boson, generically called majoron. In the early versions the majoron was an isodoublet (the imaginary part of the sneutrino) [3]; these are now ruled out by LEP measurements of the invisible $\mathrm{Z}$ width [4]. However, in the modern variants of supersymmetric models with spontaneous $\mathrm{R}$-parity breaking the majoron is a singlet (such as a right-handed sneutrino) and therefore can not be produced in the decays of the Z. Such $\left(R_{p}\right)$ models are both theoretically attractive and phenomenologically viable [5, 6]. However, quite generally study of broken R-parity phenomenology has received a lot of attention recently [7, 8, 9, 10].

Especially interesting in view of the mounting evidence for non-zero neutrino masses [11, 12, 13 is the fact that $\not_{p}$ models allow for finite - and in principle calculable - neutrino masses, and moreover could even explain their observed smallness without invoking some high-energy scale physics [14, like one does for example in the see-saw mechanism [15].

Neutrinoless double beta $\left(\beta \beta_{0 \nu}\right)$ decay is a $\Delta L=2$ process and therefore one naturally expects it to occur in models with lepton number violation in the Lagrangian. Even though there is a variety of mechanisms inducing $\beta \beta_{0 \nu}$ decay in gauge theories, one can show that whatever the leading mechanism is at least one of the neutrinos will be a Majorana particle [16], as illustrated in the black-box diagram of Fig. 1. This well-known argument establishes a deep connection between Majorana neutrino masses and $\beta \beta_{0 \nu}$ decay: in gauge theories one can not occur without the other being present. The same

remains true in supersymmetric theories [17], where moreover one can show 
that also the supersymmetric partner of the neutrino must have a $B-L$ violating Majorana-like mass term, if a Majorana mass of the neutrino exists [17]. Turning the argument around, one expects that the observed absence of $\beta \beta_{0 \nu}$ decay allows to derive stringent limits on $\not R_{p}$ parameters. This has been shown for models with explicit trilinear R-parity breaking in [18, 19]. In the current work we will concentrate on the bilinear $\not R_{p}$ terms.

$\beta \beta_{0 \nu}$ decay within supersymmetric models with bilinear R-parity breaking has been considered previously in [20]. In our derivation the final expression of the decay rate (and the corresponding nuclear matrix elements), however, differ from the one given in [20], details are given in section 3.

In an exactly supersymmetric and $S U(2)_{L}$-invariant world R-parity Violation (RPV) can be moved around the Lagrangian by field redefinitions [2]. This observation lead to the claim, that the bilinear RPV terms are unphysical and should be discarded. However it has been shown that in realistic softly broken supersymmetric models it is in general impossible to eliminate the bilinear terms [21]. Moreover, as already stated, it is possible that R-parity is a symmetry (maybe approximate) at the Lagrangian level, broken only by the ground state [5]. Such scenarios lead only to $\not L$ being therefore consistent with baryon number conservation. Models with bilinear terms can be imagined as a truncated version of the full dynamical models [21] and as such offer a systematic way to investigate RPV at the phenomenological level.

This paper is organized as follows. In the next section we introduce our conventions and notations. Section 3 derives the effective Lagrangian of $\beta \beta_{0 \nu}$ decay in $R_{p}$ SUSY with bilinear terms, while section 4 summarizes the necessary nuclear physics input. Section 5 then analyzes the constraints $\beta \beta_{0 \nu}$ decay can put on bilinear $\mathbb{R}_{p}$ parameters.

\section{Supersymmetry with explicit R-parity break- ing terms}

In this section, we set up our definitions and notation. Our conventions are usually such that in the limit of vanishing $\not R_{p}$ parameters the MSSM notation of [1] is recovered.

In the presence of bilinear R-parity and lepton number violation there is no distinction between the lepton doublet and the Higgs doublet superfield giving mass to the down-type quarks. This fact can be accounted for by defining a superfield $\hat{\Phi}$ as 


$$
\hat{\Phi}=\left(\hat{H}_{1}, \hat{L}_{1}, \hat{L}_{2}, \hat{L}_{3}\right) .
$$

For the MSSM field content the most general gauge invariant form of the renormalizable superpotential can then be written as

$$
W=\epsilon_{a b}\left[\lambda_{e}^{I J k} \hat{\Phi}_{I}^{a} \hat{\Phi}_{J}^{b} \hat{E}_{k}^{C}+\lambda_{d}^{I j k} \hat{\Phi}_{I}^{a} \hat{Q}_{j}^{b} \hat{D}_{k}^{C}+h_{u}^{j k} \hat{Q}_{j}^{a} \hat{H}_{2}^{b} \hat{U}_{k}^{C}+\mu^{I} \hat{\Phi}_{I}^{a} \hat{H}_{2}^{b}\right] .
$$

Here, $\hat{Q}$ and $\hat{D}^{C}, \hat{U}^{C}$ are the quark doublet and singlets superfields, respectively, $\hat{E}^{C}$ is the lepton singlet superfield and $\hat{H}_{2}$ the Higgs superfields with $Y\left(\hat{H}_{2}\right)=1$ responsible for the up-type quark masses, with $h_{u}^{j k}$ being the corresponding Yukawa couplings. The indices $j, k=1,2,3$ denote generations, whereas $I, J=0,1,2,3$. The indices $a, b$ are $S U(2)$ indices. In the basis (1) one can separate $W$ into an R-parity conserving and an R-parity violating $\left(\mathbb{R}_{p}\right.$ ) part

$$
W=W_{R_{P}}+W_{R_{p}},
$$

where the $R_{P}$ part is simply given by taking $I$ or $J$ to be zero and $i, j$ respectively. Then $\lambda_{e}^{0 j k}=\frac{1}{2} h_{u}^{j k}$ and $\lambda_{d}^{0 j k}=h_{d}^{j k}$. The $R_{p}$ part contains the remaining components,

$$
W_{\not R_{p}}=\lambda_{e}^{i j k} \hat{L}_{i} \hat{L}_{j} \hat{E}_{k}^{C}+\lambda_{d}^{i j k} \hat{L}_{i} \hat{Q}_{j} \hat{D}_{k}^{C}+\epsilon_{i} \hat{L}_{i} \hat{H}_{2}
$$

where $\mu_{i} \rightarrow \epsilon_{i}$, to make contact with the notation of [21]. If $W_{\mathbb{R}_{p}}$ would be the only source of RPV one could easily rotate the field $\hat{\Phi}$ into a basis $\hat{\Phi}^{\prime}$ with $\mu^{I^{\prime}}=\left(\mu^{\prime}, 0,0,0\right)$ effectively eliminating the bilinear terms. However, another source of RPV is found in the soft supersymmetry breaking part of the scalar potential. It contains the terms:

$$
\begin{aligned}
V_{R_{p}}^{s o f t} & =\tilde{A}_{e}^{I J k} \tilde{\Phi}_{I} \tilde{\Phi}_{J} \tilde{E}_{k}^{C}+\tilde{A}_{d}^{I j k} \tilde{\Phi}_{I} \tilde{Q}_{j} \tilde{D}_{k}^{C}+B^{I} \tilde{\Phi}_{I} H_{2} \\
& +\left(m_{I J}^{2}+\mu_{I} \mu_{J}\right) \tilde{\Phi}_{I} \tilde{\Phi}_{J}^{\dagger}+\cdots+\text { h.c. }
\end{aligned}
$$

where the dots represent terms not interesting for the discussion here. Rotating the superpotential as discussed above, it is easy to see that as long as the $B^{I}$ are not exactly parallel to the $\mu^{I}$ the effects of the bilinear terms of the superpotential in the rotated basis will reappear in the soft SUSY breaking terms.

Let us briefly discuss the appearance of bilinear terms $B^{I} \Phi_{I} H_{2}$. The presence of these terms imply that the tadpole equations for the sneutrinos are non-trivial, i.e. the VEVs of the sneutrino fields in general are non-zero [22]. 
As a result the MSSM vertices $\tilde{Z} \nu \tilde{\nu}$ and $\tilde{W}$ e $\tilde{e}$ create gaugino-lepton mixing mass terms $\tilde{Z} \nu<\tilde{\nu}>$ and $\tilde{W} e<\tilde{\nu}>$. This mixing then leads in the physical mass eigenstate basis to lepton number violating (LNV) interactions. The LNV vertices relevant to $\beta \beta_{0 \nu}$ decay will be discussed in the next section. The mass eigenstate fields are defined from the weak eigenstates by means of an orthogonal transformation,

$$
\begin{gathered}
\Psi_{0, i}=\Xi_{i j} \Psi_{0, j}^{\prime}, \\
\Psi_{( \pm) i}=\Delta_{i j}^{ \pm} \Psi_{( \pm) j}^{\prime},
\end{gathered}
$$

with the weak basis states in two component notation defined as

$$
\begin{gathered}
\Psi_{0}^{\prime T}=\left(\psi_{L_{1}}^{1}, \psi_{L_{2}}^{1}, \psi_{L_{3}}^{1},-i \lambda^{\prime},-i \lambda_{3}, \psi_{H_{1}}^{1}, \psi_{H_{2}}^{2}\right), \\
\Psi_{(-)}^{\prime T}=\left(\psi_{L_{1}}^{2}, \psi_{L_{2}}^{2}, \psi_{L_{3}}^{2},-i \lambda_{-}, \psi_{H_{1}}^{2}\right), \\
\Psi_{(+)}^{\prime T}=\left(\psi_{R_{1}}^{c}, \psi_{R_{2}}^{c}, \psi_{R_{3}}^{c},-i \lambda_{+}, \psi_{H_{2}}^{1}\right),
\end{gathered}
$$

where $\psi_{L_{i}}^{1}\left(\psi_{L_{i}}^{2}\right)$ are the neutrino (charged lepton) fields, $-i \lambda^{\prime}$ and $-i \lambda_{3}, \lambda_{-}$ are the unmixed photino and gaugino states, respectively, and $\psi_{H_{1,2}}^{1,2}$ are the neutral components of the Higgs doublets, and the Higgs and lepton fields are written in the original basis $\Phi$.

The mixing matrices $\Xi$ and $\Delta^{ \pm}$diagonalize the neutrino-neutralino and charged lepton-chargino mass matrices. Details are given in the appendix. We note in passing that the neutral mass matrix has such a texture that only one neutrino gains a tree-level mass, even if all of the $\epsilon_{i}$ are non-zero. The degeneracy of the remaining two neutrinos, massless at tree-level, is however lifted at the 1-loop level [23].

\section{Effective Lagrangian of $\beta \beta_{0 \nu}$ decay}

Here we derive the effective Lagrangian describing $\beta \beta_{0 \nu}$ decay in the RPV model. Formally one can define the amplitude for $\beta \beta_{0 \nu}$ decay as,

$$
R_{\beta \beta_{0 \nu}}=\left\langle(A, Z+2), 2 e^{-}\left|\mathcal{T}\left[\exp \left(i \int d^{4} x \mathcal{L}_{e f f}(x)\right)\right]\right|(Z, A)\right\rangle .
$$

For the derivation of $\mathcal{L}_{\text {eff }}$ it is convenient to decompose the different $\not_{p}$ contributions as shown in figure 2 . As indicated in the figure, in the $\not R_{p}$ MSSM the effective lepton number violating $\beta \beta_{0 \nu}$ decay amplitude arises either by the $\Delta L=2$ (massive) Majorana neutrino propagator (fig. 2.a), or through shortdistance four- and six-fermion lepton number violating vertices (fig. 2.b,c). 
The latter appear in this model in two ways, either directly from the trilinear parameters of the superpotential eq. (田), or indirectly from the bilinear terms, due to the neutrino-neutralino and chargino-charged lepton mixing.

From the superpotential one can read off the following $\Delta L=1$ trilinear terms which are relevant for $\beta \beta_{0 \nu}$ decay,

$$
\begin{aligned}
\mathcal{L}_{R_{p}}^{(T r i l i n e a r)} & =\left(\lambda_{d}^{i j k}\right)^{*}\left\{\left(\bar{\nu}_{i} P_{R} d_{k}\right) \tilde{d}_{j, L}^{*}-\left(\bar{u}_{j} P_{R} d_{k}\right) \tilde{e}_{i, L}^{*}-\left(\bar{e}_{i} P_{R} d_{k}\right) \tilde{u}_{j, L}^{*}\right. \\
& \left.-\left(\bar{u}_{j} P_{R} e_{i}^{C}\right) \tilde{d}_{k, R}\right\}+\lambda_{d}^{i j k}\left(\bar{\nu}_{i}^{C} P_{L} d_{j}\right) \tilde{d}_{k, R}^{*}+\text { h.c. }
\end{aligned}
$$

As discussed above, the bilinear terms induce mixing between the SM leptons and the MSSM charginos and neutralinos in the mass-eigenstate basis, where one finds the following $\Delta L=1$ terms [24],

$$
\begin{aligned}
\mathcal{L}_{R_{p}}^{(\text {Bilinear })}= & -\quad \frac{g}{\sqrt{2}} \kappa_{n} W_{\mu}^{-}\left(\bar{e} \gamma^{\mu} P_{L} \chi_{n}^{0}\right)+ \\
& \sqrt{2} g \quad\left\{\beta_{k}^{u}\left(\bar{\nu}_{k} P_{R} u^{C}\right) \tilde{u}_{L}+\beta_{k}^{d}\left(\bar{\nu}_{k} P_{R} d\right) \tilde{d}_{R}^{*}+\beta_{k i}^{e}\left(\bar{\nu}_{k} P_{R} e^{C}\right) \tilde{e}_{L i}\right. \\
& \left.+\beta^{c}\left(\bar{u} P_{R} e^{C}\right) \tilde{d}_{L}\right\}+ \text { h.c. }
\end{aligned}
$$

The first term in eq. (13) is generated from the Standard Model $W$-bosonfermion-fermion and R-parity-conserving interactions of the type $W-\chi^{0}-\chi^{ \pm}$ present in the MSSM. The R-parity-violating terms arise from the $\chi^{ \pm}$-fermionsfermion and $\chi^{0}$-fermion-sfermion interactions.

The coefficients in eq. (13) are defined as follows,

$$
\begin{gathered}
\kappa_{n}=\sum_{k=1}^{3} \Delta_{1 k}^{-} \Xi_{n+3, k}^{*}+\sqrt{2} \Delta_{14}^{-} \Xi_{n+3,5}^{*}+\Delta_{15}^{-} \Xi_{n+3,6}^{*} \\
\beta_{k}^{d}=-\frac{1}{3} \tan \theta_{W} \Xi_{k, 4} \\
\beta_{k}^{u}=-\frac{1}{6}\left(\tan \theta_{W} \Xi_{k, 4}+3 \Xi_{k, 5}\right) \\
\beta_{k i}^{e}=-\frac{1}{\sqrt{2}} \Delta_{14}^{-} \Xi_{i, k}+\frac{1}{2}\left(\tan \theta_{W} \Xi_{k, 4}+\Xi_{k, 5}\right) \delta_{i 1} \\
\beta^{c}=-\frac{1}{\sqrt{2}} \Delta_{14}^{-} .
\end{gathered}
$$

Indices $i, k$ above run from 1 to 3 , whereas the index $n=1,2,3,4$. 
If the $\not R_{p}$ parameters are smaller than the other typical supersymmetric parameters, one can find an approximate analytic diagonalization for the rotation matrices $\Xi$ and $\Delta^{ \pm}$using the method introduced in ref. [25]. This was done in ref. [26] and the results are presented in the appendix. In the analysis presented later on we will use this convenient approximative method and justify its use a posteriori. In leading order in the expansion parameters $\xi$ and $\xi_{L}$ the coefficients defined above are given by,

$$
\begin{gathered}
\kappa_{n} \approx \sum_{m=1}^{4} N_{n m}^{*} \xi_{1 m}^{*}-\sqrt{2}\left(\xi_{L}\right)_{11} N_{n 2}^{*}-\left(\xi_{L}\right)_{12} N_{n 3}^{*} \\
\beta_{k}^{d} \approx \frac{1}{3} \tan \theta_{W} \sum_{j=1}^{3}\left(V_{\nu}^{*}\right)_{j k} \xi_{j 1}^{*} \\
\beta_{k}^{u} \approx \frac{1}{6} \sum_{j=1}^{3}\left(V_{\nu}^{*}\right)_{j k}\left(\tan \theta_{W} \xi_{j 1}^{*}+3 \xi_{j 2}^{*}\right) \\
\beta_{k i}^{e} \approx \frac{1}{\sqrt{2}}\left(\xi_{L}\right)_{11}\left(V_{\nu}^{*}\right)_{k i}-\frac{1}{2} \sum_{j=1}^{3}\left(V_{\nu}^{*}\right)_{j k}\left(\tan \theta_{W} \xi_{j 1}^{*}+\xi_{j 2}^{*}\right) \delta_{i 1} \\
\beta^{c} \approx \frac{1}{\sqrt{2}}\left(\xi_{L}\right)_{11}
\end{gathered}
$$

In the derivation we have always neglected terms which are of higher power in the neutrino mass and terms which are suppressed by factors of $m_{u, d, e} / M_{S U S Y}$ due to left-right sfermion mixing and/or higgsino couplings. Here, $m_{u, d, e}$ are the masses of the up, down quarks and the electron mass and $M_{S U S Y}$ is the typical mass scale of SUSY particles, assumed to be of order of $\mathcal{O}(100 \mathrm{GeV})$.

In addition to the lepton number violating Lagrangians eq. (12) and eq. (13) for the calculation of $\mathcal{L}_{\text {eff }}$ for the $\beta \beta_{0 \nu}$ decay the ordinary Standard Model (SM) charged-current interaction as well as the MSSM gluino-quark-squark interaction

$$
\mathcal{L}_{\tilde{g}}=-\sqrt{2} g_{3} \frac{\lambda_{\alpha \beta}^{(a)}}{2}\left(\bar{q}_{\alpha} P_{R} \tilde{g}^{(a)} \tilde{q}_{L}^{\beta}-\bar{q}_{\alpha} P_{L} \tilde{g}^{(a)} \tilde{q}_{R}^{\beta}\right)+\text { h.c. }
$$

are needed. Here $\lambda_{\alpha \beta}^{(a)}$ are Gell-Mann matrices $(a=1, \ldots, 8)$, and the superscripts $\alpha$ and $\beta$ are color indices. 
The leading order diagrams are shown in fig. 3. The first diagram, fig. 3.a is nothing but the well-known mass mechanism, supplemented by a contribution from neutralino exchange 1 . Since for finite values of the bilinear parameters one neutrino acquires a tree-level mass this contribution is always present in the bilinear $\not R_{p}$ model. The diagram fig. 3.b represents a short-range contribution to $\beta \beta_{0 \nu}$ decay induced by the non-zero chargino-electron mixing. The remaining two diagrams are of long-range type, and involve the standard Dirac-type $(\Delta L=0)$ neutrino propagator. Note, that using the above approximations these diagrams are always proportional to the product of a bilinear times a trilinear parameter. However, these diagrams are also inherent to the model, even in the basis where the trilinear parameters are set to zero. Their contributions are, however, suppressed by a factor of $m_{f} / m_{\tilde{f}}$ from left-right sfermion mixing, which should naturally be very small for the first generation. The diagrams in fig. 3 do not include those proportional to $\left(\lambda_{d}^{i j k}\right)^{2}$. These have been previously analyzed in 18 .

Integrating out the heavy fields and carrying out a Fierz transformation, one obtains finally the effective Lagrangian

$$
\begin{aligned}
\mathcal{L}_{e f f}(x) & =\sqrt{2} G_{F}\left(V_{\nu}\right)_{1 k}\left(\bar{e} \gamma_{\mu} P_{L} \nu_{k}\right) J^{\mu} \\
& +\frac{G_{F}^{2}}{2 m_{p}}\left(\eta_{\tilde{g}}+\eta_{\chi}\right) J^{\mu} J_{\mu}\left(\bar{e} P_{R} e^{c}\right)- \\
& -\sqrt{2} G_{F}\left(\eta_{\lambda}^{(k)}\left(\bar{\nu}_{k} P_{R} e^{c}\right) J+\eta_{\lambda, q}^{(k)}\left(\bar{\nu}_{k} \sigma_{\mu \nu} P_{R} e^{c}\right) J^{\mu \nu}\right)
\end{aligned}
$$

The first term in the above equation is the usual SM charged-current interaction. Color-singlet hadronic currents in eq. (25) are given by

$$
\begin{gathered}
J=\bar{u}^{\alpha} P_{R} d_{\alpha}, \\
J^{\mu}=\bar{u}^{\alpha} \gamma_{\mu} P_{L} d_{\alpha}, \\
J^{\mu \nu}=\bar{u}^{\alpha} \sigma^{\mu \nu} P_{R} d_{\alpha} .
\end{gathered}
$$

In eq. (25) we have introduced effective lepton number violating parameters $\eta$ defined by,

\footnotetext{
${ }^{1}$ Somewhat arbitrarily we call the 4 heavy neutral states neutralinos, while the light states are referred to as neutrinos. This is done, since in the limit of vanishing $\not_{p}$ parameters the MSSM neutrino and neutralino states are recovered.
} 


$$
\begin{gathered}
\eta_{\tilde{g}}=\left(\frac{4 \pi \alpha_{S}}{9}\right)\left(\frac{4 \pi \alpha_{2}}{G_{F}^{2} m_{\tilde{d}_{L}}^{4}}\right)\left(\frac{m_{p}}{m_{\tilde{g}}}\right)\left(\beta^{c}\right)^{2} \\
\eta_{\chi}=\sum_{i=1}^{4} \frac{m_{p}}{m_{\chi_{i}^{0}}} \kappa_{i}^{2}=: \frac{m_{p}}{\left\langle m_{\chi}\right\rangle} \\
\eta_{\lambda}^{(k)}=\frac{g \lambda_{d}^{i 11}}{2 G_{F}}\left\{2 \frac{\beta_{k i}^{e}}{m_{\tilde{e}_{L, i}}^{2}}-\frac{\beta_{k}^{u}}{m_{\tilde{u}_{L}}^{2}} \delta_{i 1}-\frac{\beta_{k}^{d}}{m_{\tilde{d}_{R}}^{2}} \delta_{i 1}+\frac{\beta^{c}}{m_{\tilde{d}_{L}}^{2}} \delta_{i k}\right\} \\
\eta_{\lambda, q}^{(k)}=\frac{g \lambda_{d}^{i 11}}{8 G_{F}}\left\{\frac{\beta_{k}^{u}}{m_{\tilde{u}_{L}}^{2}} \delta_{i 1}-\frac{\beta_{k}^{d}}{m_{\tilde{d}_{R}}^{2}} \delta_{i 1}+\frac{\beta^{c}}{m_{\tilde{d}_{L}}^{2}} \delta_{i k}\right\}
\end{gathered}
$$

Here, $G_{F}$ is the Fermi constant and $m_{p}$ is the proton mass.

At this point, we would like to discuss some differences between our calculation and an earlier work [20]. In ref. [20] it was found that the shortranged part due to gluino exchange is proportional to pseudo-scalar and tensor hadronic currents. (This would imply an important enhancement of the gluino diagram due to the pion exchange mechanism first discussed for the case of trilinear R-parity breaking in [19].) However, we find that the gluinomediated diagram is proportional to the same hadronic structure as appears in the heavy Majorana neutrino exchange, characteristic of left-right symmetric models [27, 28]. Therefore, we conclude that the gluino contribution is negligibly small. Also in the long-range part of the effective Lagrangian, eq. (25), the last term in eq. (31) is missing in the corresponding equation in ref. [20], and the long-range tensor contribution proportional to $\eta_{\lambda, q}^{(k)}$ has been neglected compared to the long-range scalar-pseudoscalar part in [20].

The effective Lagrangian given above is defined in terms of quark currents. For $\beta \beta_{0 \nu}$ decay we have to reformulate $\mathcal{L}_{\text {eff }}$ in terms of nucleon currents and apply the proper nuclear wave functions to describe nucleon states in the nucleus. This is done in the following section.

\section{Half-life of $\beta \beta_{0 \nu}$ decay and definitions of nu- clear matrix elements}

Having specified the effective Lagrangian eq. (25) the derivation of the halflife of double beta decay is rather straightforward. Following the standard procedure described in [18, 30], after lengthy manipulations one finds, 


$$
\left(T_{1 / 2}^{\beta \beta_{0 \nu}}\right)^{-1}=G_{01}\left(\mathcal{M}_{\nu}\right)^{2}\left|\eta^{\Delta L=2}\right|^{2} .
$$

Here, $G_{01}$ is the leptonic phase space integral, numerical values can be found in [27], $\mathcal{M}_{\nu}$ is the nuclear matrix element governing the well-known mass mechanism of double beta decay and

$$
\eta^{\Delta L=2}=\frac{\left\langle m_{\nu}\right\rangle}{m_{e}}+\left(\frac{m_{P}}{\left\langle m_{\chi}\right\rangle}+\eta_{\tilde{g}}\right) \mathcal{R}_{N}+\eta_{\lambda} \mathcal{R}_{\lambda}+\eta_{\lambda, q} \mathcal{R}_{\lambda, q}
$$

where $\mathcal{R}_{j}=\mathcal{M}_{j} / \mathcal{M}_{\nu}$ with $j=\lambda, \lambda q, N$.

Individual nuclear matrix elements are defined by,

$$
\begin{gathered}
\mathcal{M}_{\nu}=\left(M_{G T, m}-\left(\frac{g_{V}}{g_{A}}\right)^{2} M_{F, m}\right), \\
\mathcal{M}_{N}=\left(M_{G T, N}-\left(\frac{g_{V}}{g_{A}}\right)^{2} M_{F, N}\right), \\
\mathcal{M}_{\lambda}=\alpha_{1}\left(\frac{1}{3} M_{G T^{\prime}}+M_{T^{\prime}}\right), \\
\mathcal{M}_{\lambda, q}=\left(-\frac{2}{3} \alpha_{2} M_{G T^{\prime}}+\alpha_{3} M_{F^{\prime}}+\alpha_{2} M_{T^{\prime}}\right) .
\end{gathered}
$$

The coefficients $\alpha_{i}$ are given by 29

$$
\begin{gathered}
\alpha_{1}=\frac{F_{P}^{(3)}}{\left(R m_{e}\right) g_{A}} \\
\alpha_{2}=\frac{4 T_{1}^{(3)} g_{V}\left(1-2 m_{p}\left(g_{W} / g_{V}\right)\right)}{g_{A}^{2}\left(R m_{e}\right)} \\
\alpha_{3}=\frac{4\left(2 \hat{T}_{2}^{(3)}-T_{1}^{(3)}\right) g_{V}}{g_{A}^{2}\left(R m_{e}\right)}
\end{gathered}
$$

with $g_{A} \simeq 1.26, g_{V} \simeq 1$ and $\left(g_{W} / g_{V}\right) \simeq-3.7 /\left(2 m_{P}\right)$ from CVC, while numerical values for $T_{i}^{(3)}$ can be found in ref. [31]. $m_{e}$ and $R$ are the electron mass and the nuclear radius. The matrix elements in the closure approximation are (summation over nucleon indices are suppressed) given by 32, 29]

$$
\begin{gathered}
M_{G T, k}=\left\langle 0_{f}^{+}\left|h_{k}\left(\overrightarrow{\sigma_{a}} \overrightarrow{\sigma_{b}}\right) \tau_{a}^{+} \tau_{b}^{+}\right| 0_{i}^{+}\right\rangle \\
M_{F, k}=\left\langle 0_{f}^{+}\left|h_{k} \tau_{a}^{+} \tau_{b}^{+}\right| 0_{i}^{+}\right\rangle
\end{gathered}
$$




$$
M_{T^{\prime}}=\left\langle 0_{f}^{+}\left|h_{T^{\prime}}\left\{\left(\overrightarrow{\sigma_{a}} \hat{\vec{r}}_{a b}\right)\left(\overrightarrow{\sigma_{b}} \hat{\vec{r}}_{a b}\right)-\frac{1}{3}\left(\vec{\sigma}_{a} \vec{\sigma}_{b}\right)\right\} \tau_{a}^{+} \tau_{b}^{+}\right| 0_{i}^{+}\right\rangle
$$

with $k=m, R, N$ and $h_{k}$ and $h_{T^{\prime}}$ are neutrino potentials defined as

$$
\begin{gathered}
h_{m}=\frac{2}{\pi} R \int_{0}^{\infty} d q q^{2} \frac{j_{0}\left(q r_{a b}\right) f^{2}\left(q^{2}\right)}{\omega(\omega+\bar{E})} \\
h_{R}=\frac{2}{\pi} \frac{R^{2}}{m_{P}} \int_{0}^{\infty} d q q^{4} \frac{j_{0}\left(q r_{a b}\right) f^{2}\left(q^{2}\right)}{\omega(\omega+\bar{E})} \\
h_{T^{\prime}}=\frac{2}{\pi} \frac{R^{2}}{m_{P}} \int_{0}^{\infty} d q q^{2} \frac{f^{2}\left(q^{2}\right)}{\omega(\omega+\bar{E})}\left\{q^{2} j_{0}\left(q r_{a b}\right)-3 \frac{q}{r_{a b}} j_{1}\left(q r_{a b}\right)\right\} . \\
h_{N}\left(x_{A}\right)=\frac{4 \pi m_{A}^{8}}{m_{p} m_{e}} r_{a b} \int \frac{d^{3} \mathbf{q}}{(2 \pi)^{3}} \frac{1}{\left(m_{A}^{2}+\mathbf{q}^{2}\right)^{4}} e^{i \mathbf{q r}_{a b}}
\end{gathered}
$$

with $x_{A}=m_{A} r_{a b}, \omega=\sqrt{\mathbf{q}^{2}+m_{\nu}^{2}}, \bar{E}$ the mean excitation of the intermediate nuclear states and $r_{a b}$ the nucleon-nucleon distance. $f^{2}\left(q^{2}\right)$ is the nucleon form factor, usually taken to have dipole form, $j_{0}$ and $j_{1}$ are spherical Bessel functions, and $m_{A}$ is typically $m_{A}=0.85 \mathrm{GeV}$ [32].

\section{$5 \quad \beta \beta_{0 \nu}$ Decay Constraints on $R_{p}$ Parameters}

Up to now our calculation has been independent of the use of any nuclear structure model. However, for a numerical analysis we have to assume some definite numbers for the relevant nuclear matrix elements. For definiteness we will use the pn-QRPA values of the nuclear matrix elements [18, 28, 29, 32]. Note that the uncertainty of limits on the fundamental $\not R_{p}$ parameters depend only on the square roots of the uncertainties of the nuclear structure matrix elements.

In the following analysis we will use the currently most stringent limit on double beta decay

$$
T_{1 / 2}\left({ }^{76} \mathrm{Ge}\right) \geq 1.1 \times 10^{25} \mathrm{ys}
$$

found by the Heidelberg-Moscow experiment [33].

The experimental constraint eq. (49) together with the theoretical decay rate eq. (33) defines an excluded area in a complex multi-dimensional parameter space consisting of the $\not R_{p}$ parameters as well as a number of MSSM parameters. 
We have analyzed the contributions from the different Feynman diagrams, see fig. 3, and found that for typical choices of MSSM parameters the neutrino mass mechanism is always the dominant contribution. To understand this finding, let us first explicitly calculate the effective neutrino mass in the bilinear $\not R_{p}$ model, which, using the usual GUT assumption $9 M_{1}=\frac{5}{3} \tan \theta_{W}^{2} M_{2}$, is given by,

$$
\left\langle m_{\nu}\right\rangle=\sum_{j}^{\prime} U_{e j}^{2} m_{j}=\frac{2}{3} \frac{g^{2} M_{2}}{\operatorname{det}\left(\mathcal{M}_{\chi^{0}}\right)} \Lambda_{1}^{2}
$$

where $\Lambda_{1}=\epsilon_{1} v_{1}-\omega_{1} \mu$ (see appendix) and the prime indicates summation only over light fermion states (not neutralinos), and $\omega_{1}:=\left\langle\tilde{\nu}_{1}\right\rangle$.

Let us first compare the short-range contributions with the long-range (light) neutrino exchange. All of them are of the same order in RPV parameters, i.e. proportional to $\Lambda_{1}^{2}$. By naively comparing the corresponding diagrams one might think that they are of equal importance. Closer inspection, however, reveals that this is not the case, and that the short-range contributions are suppressed compared to the long-range ones. This suppression is due to nuclear physics effects. For heavy particle exchange the two quarks interacting have to come very close together, where the strong repulsive part of the nucleon-nucleon interaction is important. For point-like nucleons this contribution would be zero, so that the corresponding nuclear matrix elements become non-zero only due to the finite nucleon size. A naive estimate of the size of the short-range contributions relative to the neutrino mass contribution can be given by comparing the typical momentum scale of the nucleons, order $p_{F}$, with the typical mass scale of SUSY particles $m_{S U S Y}$ and thus is expected to be $p_{F} / m_{S U S Y} \sim 10^{-3}$. Numerically we have found that indeed the constraints on RPV parameters derived from the gluino diagram alone are weaker than the constraints derived from considering light neutrino exchange by about the above factor.

In contrast, for the long-range diagrams, fig 3.c and 3.d, an enhancement compared to the neutrino mass diagram would be naively expected. The explicit calculation, however, shows that these diagrams are proportional to $\lambda_{d}^{i 11} \Lambda_{1}$ (in a basis with non-zero trilinear terms) or proportional to $m_{f} / m_{\tilde{f}} \Lambda_{1} \simeq$ $\mathcal{O}\left(10^{(-6)}\right) \Lambda_{1}$ for first generation fermions (in a basis where $\lambda_{d}^{i 11}$ is assumed to be zero). Thus despite a huge enhancement factor of these diagrams, from the

\footnotetext{
${ }^{2}$ Although we will use this assumption in the following, our results do not sensitively depend on this choice.
} 
propagation of the neutrino a la Dirac, no useful limits on the fundamental parameters $\epsilon_{1}$ and $\omega_{1}$ can be derived from these contributions.

Fig. 4 then shows the limits on the parameters $\epsilon_{1}$ (fig. 4.a) and $\omega_{1}$ (fig. 4.b), assuming $\tan \beta=1$ as a function of the MSSM parameters $\left(M_{2}, \mu\right)$. Limits typically of the order of a few hundred keV are found, choosing $M_{2}$ and $\mu$ of the order of up to a few hundred GeV. Note, however, that the limit on $\epsilon_{1}$ depends rather strongly on the choice of $\tan \beta$, and detoriates proportional to $\tan \beta$ for large values of this parameter.

The robustness of the double beta decay bound is then demonstrated in fig. 5, where calculated half-lives for the $\beta \beta_{0 \nu}$ decay of ${ }^{76} G e$ as a function of $\left\langle\tilde{\nu}_{1}\right\rangle$ are shown. For this figure we have varied the MSSM parameters freely and randomly in a range motivated by naturalness arguments, i.e. $M_{2}$ and $\mu$ between $100 \mathrm{GeV}$ and $1 \mathrm{TeV}$ and $\tan \beta=1-50$. A clear absolute upper limit of the order of $1.5 \mathrm{MeV}$ on $\left\langle\tilde{\nu}_{1}\right\rangle$ is found from this analysis.

The limits shown in fig. 4,5 assume that only one bilinear $\not R_{p}$ parameter is non-zero, which is unnatural from the point of view of the minimization of the scalar potential [21]. A combined limit on $\left(\epsilon_{1}, \omega_{1}\right)$ is therefore shown in fig. 6., for $\mu=100 \mathrm{GeV}$, various values of $M_{2}$ and $\tan \beta=1$ (fig. 6.a) and $\tan \beta=10$ (fig. 6.b). Obviously, the limits on $\epsilon_{1}$ depend rather strongly on $\tan \beta$, whereas the limits on $\omega_{1}$ do not. Note the double logarithmic scale.

In summary we find that $\beta \beta_{0 \nu}$ decay provides very stringent limits on RPV parameters of the first generation. For any reasonable choice of MSSM parameters in the bilinear $\not R_{p}$ model the mass mechanism is the dominant contribution to $\beta \beta_{0 \nu}$ decay.

\section{Discussion and Conclusion}

We have calculated the contributions of (bilinear) R-parity breaking supersymmetry to neutrinoless double beta decay. This model mimics closely the case of spontaneous breaking of $\mathrm{R}$-parity. We find that $\beta \beta_{0 \nu}$ decay constrains only a subset of the possible bilinear parameters, namely $\epsilon_{1}$ and the sneutrino $\mathrm{VEV}$ of the first generation $\omega_{1}$. This is a general property of the theory and does not mean any fine-tuning of parameters. For the first generation $\not_{p}$ parameters, on the other hand, $\beta \beta_{0 \nu}$ decay provides very stringent limits, typically of the order of a few hundred keV up to a few MeV. With these limits at hand it seems rather hopeless to search for RPV in the first generation at colliders. From this point of view a possible interpretation of HERA events in 
terms of $R_{p}$ interactions would be rather unlikely. However, it is important to stress that, even though we find rather stringent constraints on the magnitude of first generation $R_{p}$ parameters, these do not limit in any way the attainable magnitudes of R-parity breaking signatures expected at colliders, since the latter involve mainly the third generation, i.e. they involve $\tau$ 's or $\nu_{\tau}$ 's. This fact follows very naturally, for example, in scenarios with radiative symmetry breaking, due to the larger Yukawa couplings that characterize the third generation.

In our present study we find that the main origin $\beta \beta_{0 \nu}$ decay in this model is the mass mechanism and conclude that other contributions are practically irrelevant.

\section{Acknowledgement}

We are grateful to S.G. Kovalenko for useful comments. This work was supported by the Spanish DGICYT under grant PB95-1077 and by the European Union's TMR program under grants ERBFMRXCT960090 and ERBFMBICT983000.

\section{Appendix A: Mass matrices}

\subsection{Neutral fermion mass matrix}

The presence of the lepton number violating bilinear terms in the superpotential, see eq. (3), leads to mixing of the neutralino and neutrino states [3]. We treat the effects of R-parity violation perturbatively, using the method in 25] in order to diagonalize the various mass matrices and hence determine the corresponding couplings. In doing this we follow the conventions given in ref. [26]. In two component notation let $\Psi_{0}^{\prime}$ denote the column vector of neutrinos and neutralinos in the basis

$$
\Psi_{0}^{\prime T}=\left(\psi_{L_{1}}^{1}, \psi_{L_{2}}^{1}, \psi_{L_{3}}^{1},-i \lambda^{\prime},-i \lambda_{3}, \psi_{H_{1}}^{1}, \psi_{H_{2}}^{2}\right),
$$

where $\psi_{L_{i}}^{1}$ are the neutrino fields, $-i \lambda^{\prime}$ and $-i \lambda_{3}$ are the unmixed photino and gaugino states, respectively, and $\psi_{H_{1,2}}^{1,2}$ are the neutral components of the Higgs doublets. The Lagrangian describing the neutrino/neutralino masses is then given by,

$$
\mathcal{L}_{\text {mass }}^{0}=-\frac{1}{2} \Psi_{0}^{\prime T} \mathcal{M}_{0} \Psi_{0}^{\prime}+\text { h.c. }
$$

The mass matrix can be written in a block form, 


$$
\mathcal{M}_{0}=\left(\begin{array}{cc}
0 & m \\
m^{T} & \mathcal{M}_{\chi^{0}}
\end{array}\right) .
$$

Here, the submatrix $m$ contains entries from the bilinear $\not R_{p}$ parameters,

$$
m=\left(\begin{array}{cccc}
-\frac{1}{2} g^{\prime} \omega_{e} & \frac{1}{2} g \omega_{e} & 0 & -\epsilon_{e} \\
-\frac{1}{2} g^{\prime} \omega_{\mu} & \frac{1}{2} g \omega_{\mu} & 0 & -\epsilon_{\mu} \\
-\frac{1}{2} g^{\prime} \omega_{\tau} & \frac{1}{2} g \omega_{\tau} & 0 & -\epsilon_{\tau}
\end{array}\right)
$$

$\omega_{i}:=\left\langle\tilde{\nu}_{i}\right\rangle \cdot \mathcal{M}_{\chi^{0}}$ is the MSSM neutralino mass matrix, given by,

$$
\mathcal{M}_{\chi^{0}}=\left(\begin{array}{cccc}
M_{1} & 0 & -\frac{1}{2} g^{\prime} v_{1} & \frac{1}{2} g^{\prime} v_{2} \\
0 & M_{2} & \frac{1}{2} g v_{1} & -\frac{1}{2} g v_{2} \\
-\frac{1}{2} g^{\prime} v_{1} & \frac{1}{2} g v_{1} & 0 & -\mu \\
\frac{1}{2} g^{\prime} v_{2} & -\frac{1}{2} g v_{2} & -\mu & 0
\end{array}\right) .
$$

One defines the mass eigenstates by means of the rotation

$$
\Psi_{0, i}=\Xi_{i j} \Psi_{0, j}^{\prime}
$$

The mass matrix then is diagonalize by

$$
\Xi^{*} \mathcal{M}_{0} \Xi^{\dagger}=\hat{\mathcal{M}}_{0}
$$

where $\hat{\mathcal{M}}_{0}$ is a diagonal matrix, with the eigenvalues as elements. Note that these conventions are chosen such that in the limit of vanishing $\not_{p}$ parameters the usual MSSM convention $[1], \Xi \rightarrow \mathcal{N}$, is recovered.

\subsection{Charged fermion mass matrix}

For the charged fermion sector the mass part of the Lagrangian can be written as,

$$
\mathcal{L}_{\text {mass }}^{ \pm}=-\Psi_{(-)}^{\prime T} \mathcal{M}_{ \pm} \Psi_{(+)}^{\prime}+\text { h.c. }
$$

where in two component Weyl spinor basis

$$
\begin{aligned}
& \Psi_{(-)}^{\prime T}=\left(\psi_{L_{1}}^{2}, \psi_{L_{2}}^{2}, \psi_{L_{3}}^{2},-i \lambda_{-}, \psi_{H_{1}}^{2}\right), \\
& \Psi_{(+)}^{\prime T}=\left(\psi_{R_{1}}^{c}, \psi_{R_{2}}^{c}, \psi_{R_{3}}^{c},-i \lambda_{+}, \psi_{H_{2}}^{1}\right) .
\end{aligned}
$$

The $(5 \times 5)$ charged fermion mass can be written as, 


$$
\mathcal{M}_{ \pm}=\left(\begin{array}{cc}
M_{l} & E \\
E^{\prime} & \mathcal{M}_{\chi^{ \pm}}
\end{array}\right),
$$

where $\mathcal{M}_{\chi^{ \pm}}$is the usual MSSM chargino mass matrix

$$
\mathcal{M}_{\chi^{ \pm}}=\left(\begin{array}{cc}
M & \frac{1}{\sqrt{2}} g v_{1} \\
\frac{1}{\sqrt{2}} g v_{2} & \mu
\end{array}\right),
$$

and the sub-matrices $E$ and $E^{\prime}$ give rise to chargino-charged lepton mixing. They are defined as

$$
\begin{gathered}
E=\left(\begin{array}{cc}
\frac{1}{\sqrt{2}} g \omega_{e} & \epsilon_{e} \\
\frac{1}{\sqrt{2}} g \omega_{\mu} & \epsilon_{\mu} \\
\frac{1}{\sqrt{2}} g \omega_{\tau} & \epsilon_{\tau}
\end{array}\right), \\
E^{\prime}=\left(\begin{array}{ccc}
0 & 0 & 0 \\
\frac{1}{\sqrt{2}} h_{1 i} \omega_{i} & \frac{1}{\sqrt{2}} h_{2 i} \omega_{i} & \frac{1}{\sqrt{2}} h_{3 i} \omega_{i}
\end{array}\right) .
\end{gathered}
$$

$M_{l}$ finally is the charged lepton mass matrix due to the ordinary Yukawa interactions. The rotation from the weak to the mass eigenstate basis is defined as,

$$
\Psi_{( \pm) i}=\Delta_{i j}^{ \pm} \Psi_{( \pm) j}^{\prime}
$$

where $\Delta^{ \pm}$diagonalize the mass matrix via

$$
\left(\Delta^{-}\right)^{*} \mathcal{M}_{ \pm}\left(\Delta^{+}\right)^{\dagger}=\operatorname{Diag}\left\{m_{i}^{l}, m_{\chi_{k}^{ \pm}}\right\} .
$$

$m_{i}^{l}$ are the known masses of the charged leptons, and $m_{\chi_{k}^{ \pm}}$the "chargino" masses, $k=1, \ldots, 4$.

Note, that without making assumptions about the structure of $M_{l}$, it is impossible to diagonalize $\mathcal{M}_{ \pm}$. For numerical purposes one therefore has to assume that $M_{l}$ (i.e. the Yukawa couplings $h_{i j}$ ) can be brought into a diagonal form. The constraint that the smallest 3 eigenvalues of $\mathcal{M}_{ \pm}$should coincide with the known masses of the charged leptons, can than be reformulated as a set of equations for the Yukawa couplings, which for finite $\not R_{p}$ parameters differ from the MSSM equations. 


\section{Appendix B: Approximate diagonalization of mass matrices}

\subsection{Neutral fermion mass matrix}

If the $\not R_{p}$ parameters are small in the sense that for

$$
\xi=m \cdot \mathcal{M}_{\chi^{0}}^{-1}
$$

all $\xi_{i j} \ll 1$, one can find an approximate solution for the neutrino/neutralino mass matrix $\Xi$. Explicitly, the $\xi_{i j}$ are given by

$$
\begin{gathered}
\xi_{i 1}=\frac{g^{\prime} M_{2} \mu}{2 \operatorname{det}\left(\mathcal{M}_{\chi^{0}}\right)} \Lambda_{i}, \\
\xi_{i 2}=-\frac{g M_{1} \mu}{2 \operatorname{det}\left(\mathcal{M}_{\chi^{0}}\right)} \Lambda_{i}, \\
\xi_{i 3}=\frac{\epsilon_{i}}{\mu}+\frac{\left(g^{2} M_{1}+g^{\prime 2} M_{2}\right) v_{2}}{4 \operatorname{det}\left(\mathcal{M}_{\chi^{0}}\right)} \Lambda_{i}, \\
\xi_{i 4}=-\frac{\left(g^{2} M_{1}+g^{\prime 2} M_{2}\right) v_{1}}{4 \operatorname{det}\left(\mathcal{M}_{\chi^{0}}\right)} \Lambda_{i} .
\end{gathered}
$$

It is interesting to note, that only $\xi_{i 3}$ is not proportional to $\Lambda_{i}$, where $\Lambda_{i}$ is

$$
\Lambda_{i}=\mu \omega_{i}-v_{1} \epsilon_{i} .
$$

In leading order in $\xi$ the mixing matrix $\Xi$ is given by,

$$
\begin{aligned}
\Xi^{*} & =\left(\begin{array}{cc}
V_{\nu}^{T} & 0 \\
0 & N^{*}
\end{array}\right)\left(\begin{array}{cc}
1-\frac{1}{2} \xi \xi^{\dagger} & -\xi \\
\xi^{\dagger} & 1-\frac{1}{2} \xi^{\dagger} \xi
\end{array}\right) \\
& =\left(\begin{array}{cc}
V_{\nu}^{T}\left(1-\frac{1}{2} \xi \xi^{\dagger}\right) & -V_{\nu}^{T} \xi \\
N^{*} \xi^{\dagger} & N^{*}\left(1-\frac{1}{2} \xi^{\dagger} \xi\right)
\end{array}\right)
\end{aligned}
$$

The second matrix in eq. (73) above block-diagonalize $\mathcal{M}_{0}$ approximately to the form $\operatorname{diag}\left(m_{e f f}, \mathcal{M}_{\chi^{0}}\right)$, where

$$
m_{e f f}=-m \cdot \mathcal{M}_{\chi^{0}}^{-1} m^{T}=\frac{M_{1} g^{2}+M_{2} g^{\prime 2}}{4 \operatorname{det}\left(\mathcal{M}_{\chi^{0}}\right)}\left(\begin{array}{ccc}
\Lambda_{e}^{2} & \Lambda_{e} \Lambda_{\mu} & \Lambda_{e} \Lambda_{\tau} \\
\Lambda_{e} \Lambda_{\mu} & \Lambda_{\mu}^{2} & \Lambda_{\mu} \Lambda_{\tau} \\
\Lambda_{e} \Lambda_{\tau} & \Lambda_{\mu} \Lambda_{\tau} & \Lambda_{\tau}^{2}
\end{array}\right)
$$


Here, $\operatorname{det}\left(\mathcal{M}_{\chi^{0}}\right)$ is the determinant of $\mathcal{M}_{\chi^{0}}$

$$
\operatorname{det}\left(\mathcal{M}_{\chi^{0}}\right)=-\mu^{2} M_{1} M_{2}+\frac{1}{2} \mu v_{1} v_{2}\left(M_{1} g^{2}+M_{2} g^{2}\right) .
$$

The submatrices $N$ and $V_{\nu}$ in eq. (73) diagonalize $\mathcal{M}_{\chi^{0}}$ and $m_{\text {eff }}$ in the following way:

$$
\begin{gathered}
N^{*} \mathcal{M}_{\chi^{0}} N^{\dagger}=\operatorname{diag}\left(m_{\chi_{i}^{0}}\right), \\
V_{\nu}^{T} m_{e f f} V_{\nu}=\operatorname{diag}\left(0,0, m_{\nu}\right),
\end{gathered}
$$

where $m_{\chi_{i}^{0}}$ are the "neutralino" mass eigenstates, and the only non-zero neutrino mass is given by

$$
m_{\nu}=\operatorname{Tr}\left(m_{e f f}\right)=\frac{M_{1} g^{2}+M_{2} g^{2}}{4 \operatorname{det}\left(\mathcal{M}_{\chi^{0}}\right)}|\vec{\Lambda}|^{2} .
$$

Note that this convention is chosen such that eq. (76) coincides with the standard MSSM convention by Haber and Kane [1].

For $V_{\nu}$ one can find an analytic form,

$$
V_{\nu}=\left(\begin{array}{ccc}
1 & 0 & 0 \\
0 & \cos \theta_{23} & -\sin \theta_{23} \\
0 & \sin \theta_{23} & \cos \theta_{23}
\end{array}\right) \cdot\left(\begin{array}{ccc}
\cos \theta_{13} & 0 & -\sin \theta_{13} \\
0 & 1 & 0 \\
\sin \theta_{13} & 0 & \cos \theta_{13}
\end{array}\right)
$$

i.e.,

$$
V_{\nu}=\left(\begin{array}{ccc}
\cos \theta_{13} & 0 & -\sin \theta_{13} \\
-\sin \theta_{13} \sin \theta_{23} & \cos \theta_{23} & -\cos \theta_{13} \sin \theta_{23} \\
\sin \theta_{13} \cos \theta_{23} & \sin \theta_{23} & \cos \theta_{13} \cos \theta_{23}
\end{array}\right)
$$

where the mixing angles can be expressed in terms of the "alignment vector" $\vec{\Lambda}$ as follows:

$$
\begin{gathered}
\tan \theta_{13}=-\frac{\Lambda_{e}}{\left(\Lambda_{\mu}^{2}+\Lambda_{\tau}^{2}\right)^{\frac{1}{2}}} \\
\tan \theta_{23}=\frac{\Lambda_{\mu}}{\Lambda_{\tau}}
\end{gathered}
$$




\subsection{Charged fermion mass matrix}

Similarly to the case of the neutralino mass matrix discussed above, for small values of the $\ell_{p}$ breaking parameters it is possible to find an approximate diagonalization procedure for the charged mass matrix.

Using the fact that the matrix $E^{\prime}$ is proportional to the Yukawa couplings of the charged leptons, one can assume $E^{\prime} \approx 0$.

Define then,

$$
\begin{gathered}
\xi_{L}^{*}=E\left(\mathcal{M}_{\chi^{ \pm}}\right)^{-1} \\
\xi_{R}^{*}=M_{l}^{\dagger} E \mathcal{M}_{\chi^{ \pm}}^{-1}\left(\mathcal{M}_{\chi^{ \pm}}^{-1}\right)^{T}=M_{l}^{\dagger} \xi_{L}^{*}\left(\mathcal{M}_{\chi^{ \pm}}^{-1}\right)^{T} .
\end{gathered}
$$

Note that $\xi_{R} \sim \xi_{L}\left(m_{l} / M\right)$. Explicitly,

$$
\begin{gathered}
\left(\xi_{L}^{*}\right)_{i 1}=\frac{g \Lambda_{i}}{\sqrt{2}\left(M \mu-\frac{1}{2} g^{2} v_{1} v_{2}\right)} \\
\left(\xi_{L}^{*}\right)_{i 2}=\frac{\epsilon_{i}}{\mu}-\frac{g^{2} v_{2} \Lambda_{i}}{2 \mu\left(M \mu-\frac{1}{2} g^{2} v_{1} v_{2}\right)}
\end{gathered}
$$

The rotation matrices $\Delta^{ \pm}$are then given in leading order in $\xi_{L}$ and $\xi_{R}$ as

$$
\begin{aligned}
& \left(\Delta^{-}\right)^{*}=\left(\begin{array}{cc}
V_{L} & 0 \\
0 & U^{*}
\end{array}\right)\left(\begin{array}{cc}
1-\frac{1}{2} \xi_{L}^{*} \xi_{L}^{T} & -\xi_{L}^{*} \\
\xi_{L}^{T} & 1-\frac{1}{2} \xi_{L}^{T} \xi_{L}^{*}
\end{array}\right), \\
& \left(\Delta^{+}\right)^{\dagger}=\left(\begin{array}{cc}
1-\frac{1}{2} \xi_{R}^{*} \xi_{R}^{T} & \xi_{R}^{*} \\
-\xi_{R}^{T} & 1-\frac{1}{2} \xi_{R}^{T} \xi_{R}^{*}
\end{array}\right)\left(\begin{array}{cc}
V_{R}^{\dagger} & 0 \\
0 & V^{\dagger}
\end{array}\right) .
\end{aligned}
$$

The conventions are chosen such, that in the limit of vanishing $\not R_{p}$ eqs (87), (88) simplify to the MSSM notations:

$$
\begin{aligned}
U^{*} \mathcal{M}_{\chi^{ \pm}} V^{\dagger} & =\hat{\mathcal{M}}_{\chi^{ \pm}}, \\
V_{L} M_{l} V_{R}^{\dagger} & =\hat{M}_{l},
\end{aligned}
$$

where again the hat indicates a diagonal matrix.

\section{References}

[1] For a review on the MSSM, see for example: H.E. Haber and G.L.Kane, Phys. Rep. 117 (1985) 75 
[2] Hall L. and Suzuki M., Nucl. Phys. B231 419 (1984)

[3] G. G. Ross, J. W. F. Valle. Phys.Lett. 151B 375 (1985); John Ellis, G. Gelmini, C. Jarlskog, G.G. Ross, J. W. F. Valle, Phys.Lett. 150B 142 (1985)

[4] C. S. Aulakh, R. N. Mohapatra, Phys. Lett. 119B 136, (1982); A. Santamaria, J. W. F. Valle, Phys. Lett. 195B, 423, (1987).

[5] A. Masiero and J. W. F. Valle, Phys. Lett. B251, 273 (1990); J. C. Romão, A. Ioannissyan and J. W. F. Valle, Phys. Rev. D55 (1997) 427

[6] For reviews see J.W.F. Valle, hep-ph/9603307 and hep-ph/9808292

[7] M.C. Gonzalez-Garcia and J.W.F. Valle, Nucl. Phys. B 355, 330 (1991).

[8] K. Huitu, J. Maalampi, K. Puolamaki, Eur. Phys. J. C6 (1999) 159-166; hep-ph/9705406

[9] F. de Campos, M.A. García-Jareño, A.S. Joshipura, J. Rosiek, and J.W.F. Valle, Nucl. Phys. B451, 3 (1995); A.S. Joshipura and M. Nowakowski, Phys. Rev. D 51, 2421 (1995); T. Banks, Y. Grossman, E. Nardi, and Y. Nir, Phys. Rev. D 52, 5319 (1995); F. Vissani and A.Yu. Smirnov, Nucl.Phys. B460, 37 (1996); F.M. Borzumati, Y. Grossman, E. Nardi, Y. Nir, Phys. Lett. B 384, 123 (1996); H.P. Nilles and N. Polonsky, Nucl. Phys. B484, 33 (1997); B. de Carlos, P.L. White, Phys. Rev. D 55, 4222 (1997); E. Nardi, Phys. Rev. D 55, 5772 (1997); S. Roy and B. Mukhopadhyaya, Phys. Rev. D 55, 7020 (1997); M. Carena, S. Pokorski, and C.E.M. Wagner, hep-ph/9801251; M.E. Gómez and K. Tamvakis, hep-ph/9801348.

[10] V. Barger, G. F. Giudice, and T. Han Phys. Rev. D40,2987 (1989); for a recent compilation see G. Bhattacharyya, Nucl. Phys. Proc. Suppl. 52 A, 83 (1997) and references therein.

[11] For a recent analysis of atmospheric neutrino data see M. C. GonzalezGarcia, H. Nunokawa, O. L. G. Peres, J. W. F. Valle, hep-ph/9807305; M. C. Gonzalez-Garcia, H. Nunokawa, O. L. G. Peres, T. Stanev, J. W. F. Valle, Phys. Rev. D58, 033004, (1998) hep-ph/9801368.

[12] For a recent analysis of the solar neutrino data see J.N. Bahcall, P.I. Krastev, A.Yu. Smirnov, Phys. Rev. D58, 096016, (1998). 
[13] For a recent review on the status of neutrino physics see, e.g. J. W. F. Valle, hep-ph/9809234

[14] J. C. Romão, J. W. F. Valle, Nucl. Phys. B381 (1992) 87

[15] M. Gell-Mann, P. Ramond, and R. Slansky, in Supergravity, ed. F. van Nieuwenhuizen and D.Freedman, (North Holland, Amsterdam, 1979), p.315; T. Yanagida, Proc. of the Workshop on Unified Theory and Baryon Number of the Universe, KEK, Japan, 1979; R. N. Mohapatra and G. Senjanović, Phys. Rev. D23 (1981) 165

[16] J. Schechter and J.W.F. Valle, Phys.Rev. D 25 (1982) 2951

[17] M. Hirsch, H.V. Klapdor-Kleingrothaus and S.G. Kovalenko, Phys. Lett. B398 (1997) 311; ibid B403 (1997) 291

[18] M. Hirsch, H.V. Klapdor-Kleingrothaus and S.G. Kovalenko, Phys. Rev. Lett. 75 (1995) 17; Phys. Rev. D53 (1996) 1329; Phys. Lett. B372 (1996) 181, erratum B381 (1996) 488

[19] A. Faessler, S.G. Kovalenko, F. Simkovic and J. Schwieger, Phys. Rev. Lett. 78 (1997) 183

[20] A. Faessler, S. Kovalenko, F. Simkovic, Phys.Rev. D57 (1998) 055004 hep-ph/9712535

[21] M.A. Díaz, J.C. Romão, and J.W.F. Valle, Nucl.Phys. B524 23-40 (1998), [hep-ph/9706315], for a review see J. W. F. Valle, hep-ph/9808292

[22] See discussion given in ref. [21].

[23] R. Hempfling; Nucl. Phys. B478 (1996) 3

[24] M. C. Gonzalez-Garcia, J. C. Romão, J. W. F. Valle, Nucl. Phys. B391, 100 (1993); J. C. Romão, F. de Campos, A. Garcia-Jareno, M. B. Magro, J. W. F. Valle, Nucl.Phys. B482 (1996) 3-23 hep-ph/9604244.

[25] J. Schechter, J.W.F. Valle, Phys. Rev. D25 774, (1982).

[26] M. Nowakowski and A. Pilaftsis, Nucl. Phys. B461 (1996) 19, hepph/9508271

[27] M. Doi, T. Kotani, E. Takasugi, Progr. Theor. Phys. Suppl. 83 (1985) 1 
[28] M. Hirsch, H.V. Klapdor-Kleingrothaus and O. Panella, Phys. Lett. B374 (1996) 7

[29] H. Päs, H.V.Klapdor-Kleingrothaus, M. Hirsch and S.G. Kovalenko, submitted to Phys. Lett. B

[30] M. Hirsch, H.V.Klapdor-Kleingrothaus and S.G. Kovalenko, Phys. Rev. D57 (1998) 1947

[31] S. Adler et al., Phys. Rev. D 11, (1975) 3309

[32] K. Muto, E. Bender and H.V. Klapdor, Z. Phys. A334 (1989) 177, 187

[33] L. Baudis et al., Phys. Lett. B407 (1997) 219

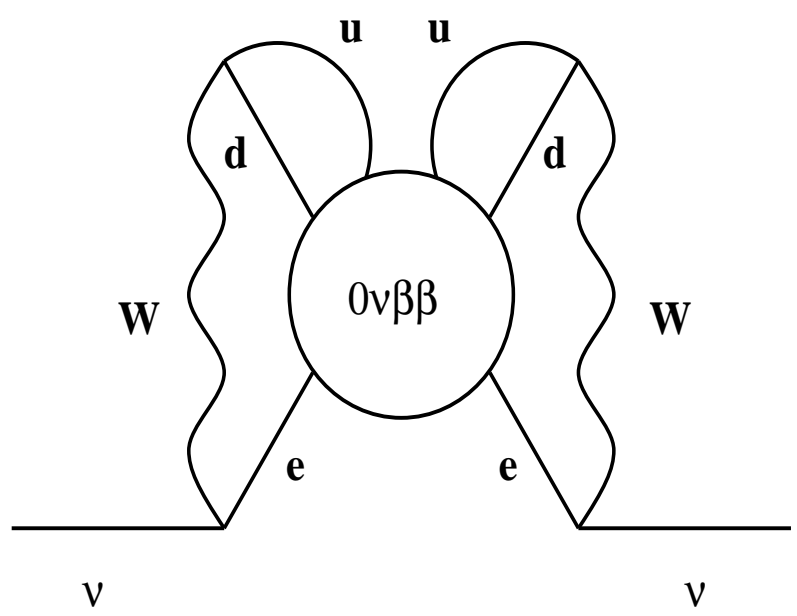

Figure 1: Diagram illustrating the connection between the Majorana mass of the neutrino and the amplitude of double beta decay. 


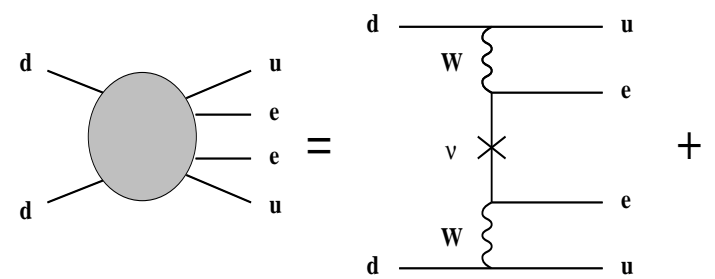

(a)

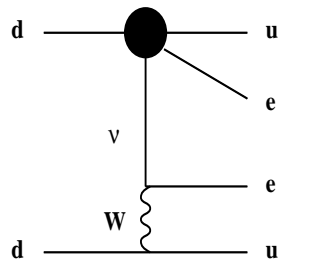

(b)

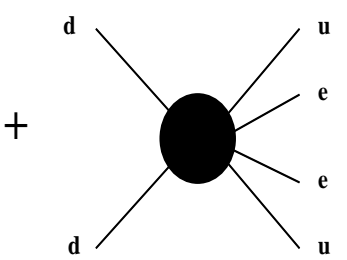

(c)

Figure 2: Decomposition of the double beta decay amplitude into long-range and short-range contributions.
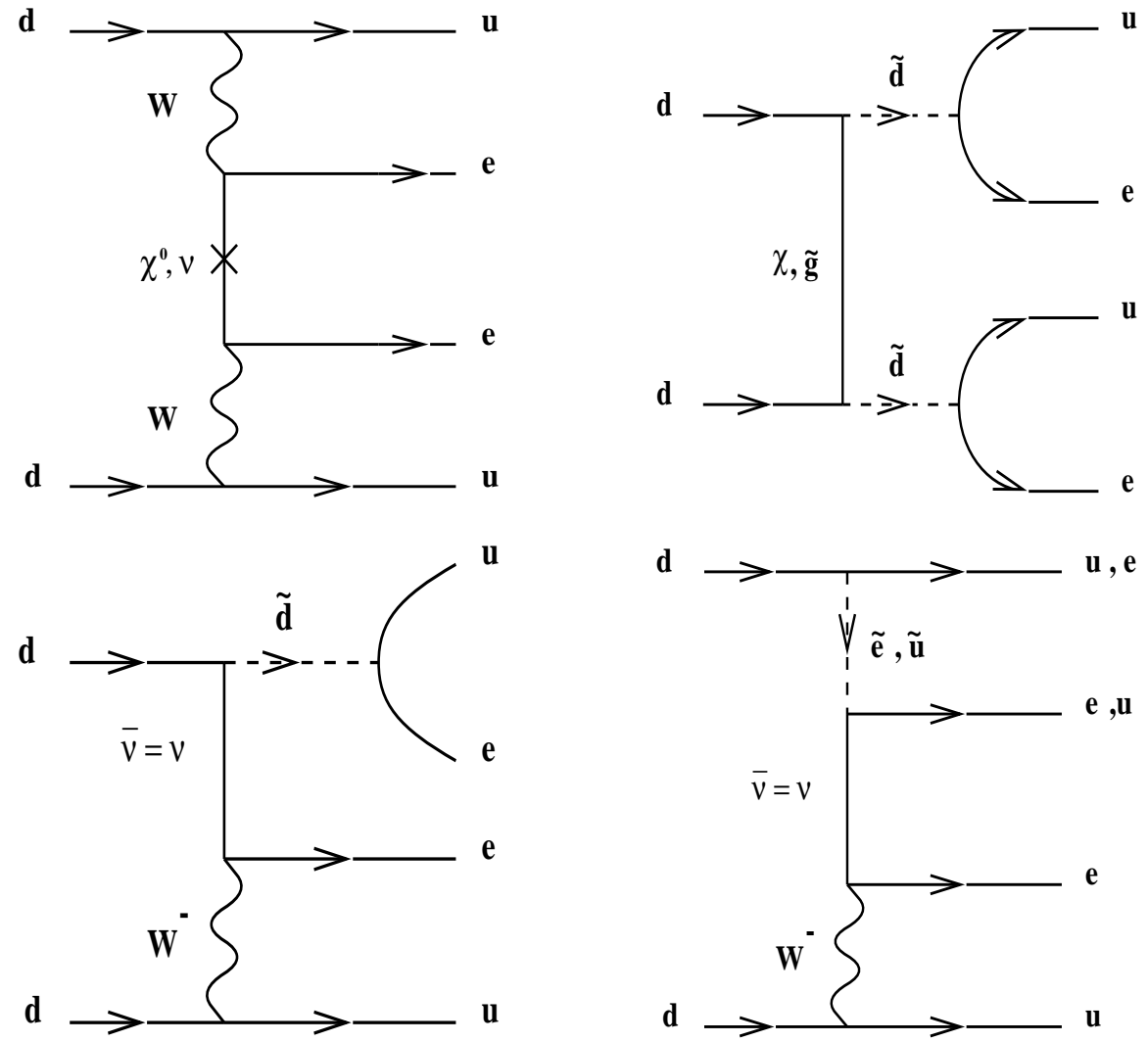

Figure 3: Leading double-beta-decay Feynman graphs in the $R_{p}$ model. 


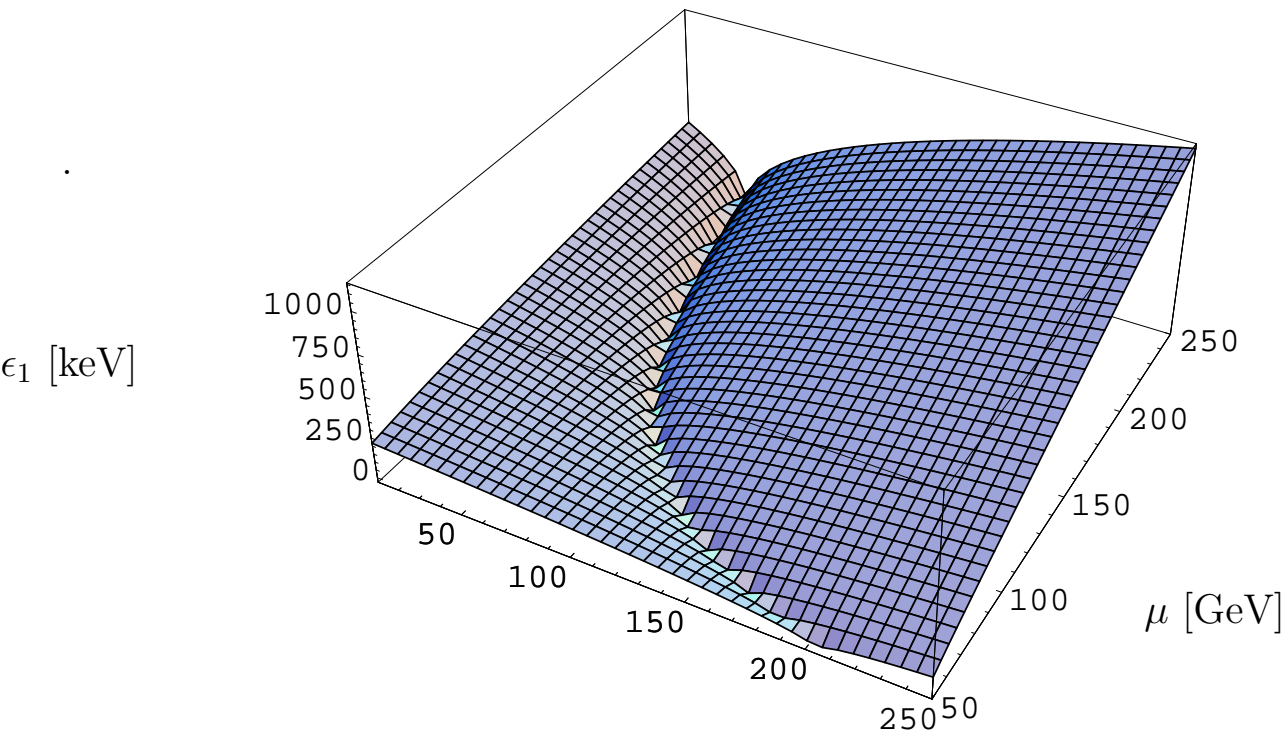

$M_{2}[\mathrm{GeV}]$

Figure 4.a: Upper limit on the individual parameter $\epsilon_{1}$ for $\tan \beta=1$ and $\omega_{i}=0$ as a function of $\left(M_{2}, \mu\right)$.

$\omega_{1}[\mathrm{keV}]$

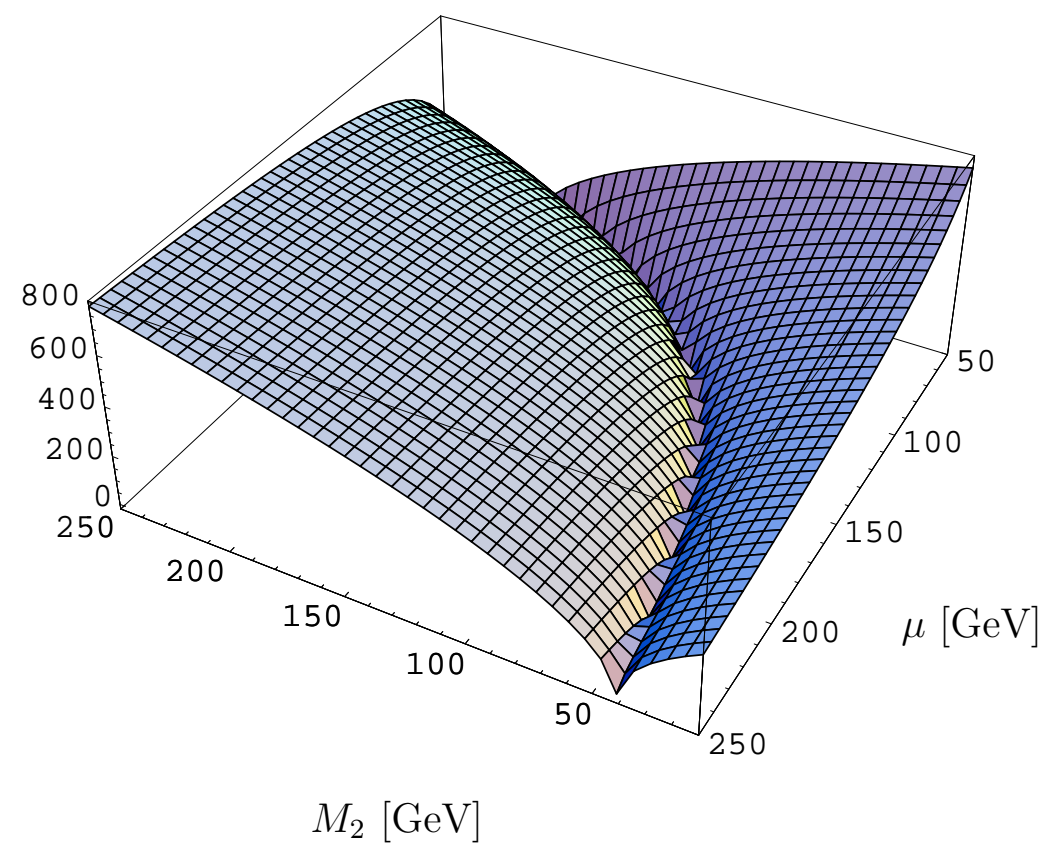

Figure 4.b: Same as figure 4.a, but for $\omega_{1}$, assuming $\epsilon_{1}=0$. 


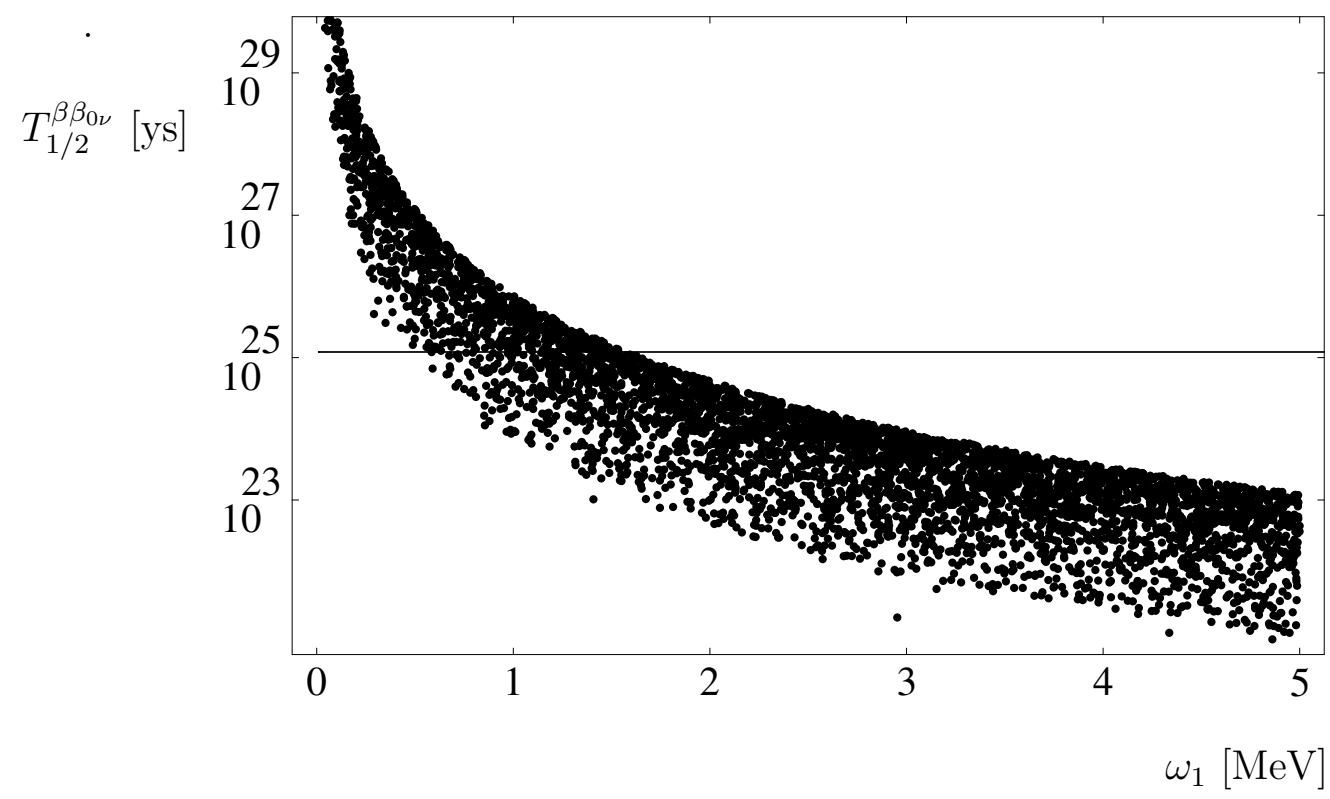

Figure 5: Calculated half-life for the $\beta \beta_{0 \nu}$ decay of ${ }^{76}$ Ge as function of $\omega_{1}$ for a random variation of the MSSM parameters, $M_{2}$ and $\mu$ from 100 GeV to 1 Te $V$ and $\tan \beta=1-50$. 


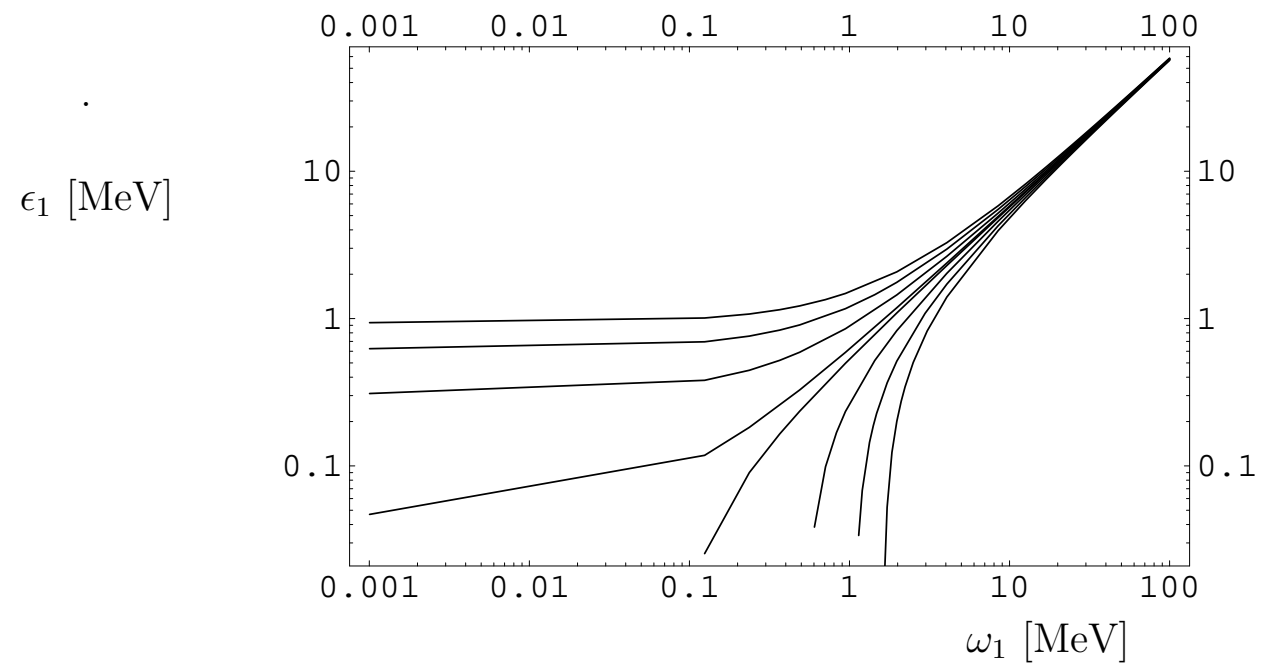

Figure 6.a: Upper limits on $\left(\epsilon_{1}, \omega_{1}\right)$ assuming $\left\langle m_{\nu}\right\rangle \leq 0.5 \mathrm{eV}, \tan \beta=1$ and $\mu=100 \mathrm{GeV}$, for different values of $M_{2}, M_{2}=100,200,500,1000 \mathrm{GeV}$. Note that the allowed range is always in between two lines of constant $M_{2}$.

$\epsilon_{1}[\mathrm{MeV}]$

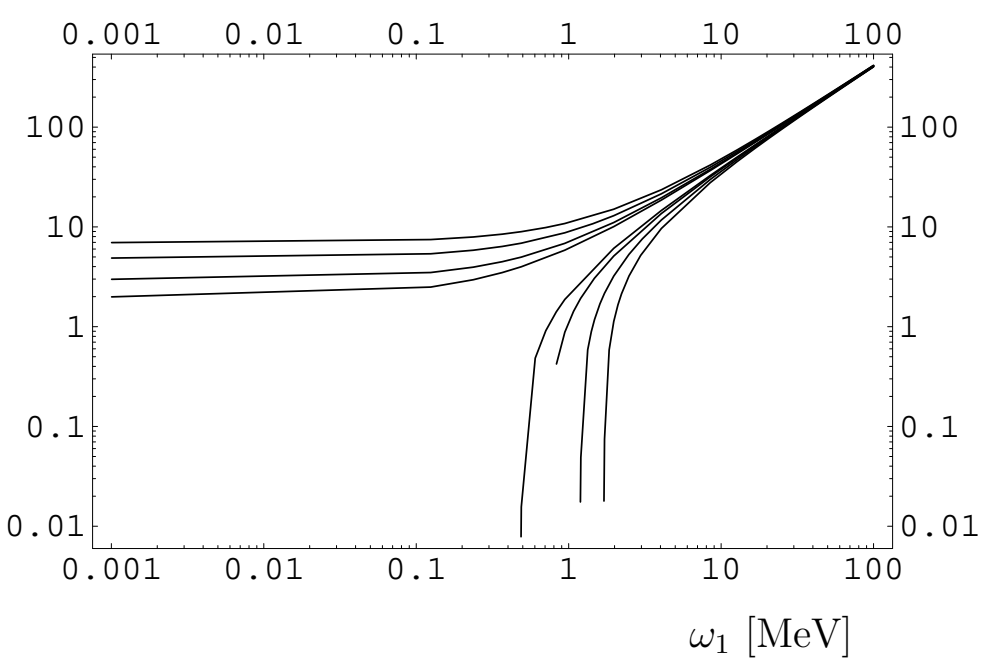

Figure 6.b: Same as figure 6.a, but for $\tan \beta=10$. 